\title{
Safety and efficacy of alteplase in the treatment of acute ischemic stroke
}

\author{
This article was published in the following Dove Press journal: \\ Vascular Health and Risk Management \\ 5 May 2009 \\ Number of times this article has been viewed
}

\section{Giuseppe Micieli \\ Simona Marcheselli \\ Piera Angela Tosi}

Neurology and Stroke Unit, IRCCS Istituto Clinico Humanitas, Rozzano (MI), Italy
Correspondence: Giuseppe Micieli IRCCS Istituto Clinico Humanitas, via Manzoni, 56, 20089 Rozzano (MI), Italy $\mathrm{Tel}+390282244698$

Fax +39028224 4693

Email giuseppe.micieli@humanitas.it

\begin{abstract}
After publication of the results of the National Institute of Neurological Disorders and Stroke study, the application of intravenous thrombolysis for ischemic stroke was launched and has now been in use for more than 10 years. The approval of this drug represented only the first step of the therapeutic approach to this pathology. Despite proven efficacy, concerns remain regarding the safety of recombinant tissue-type plasminogen activator for acute ischemic stroke used in routine clinical practice. As a result, a small proportion of patients are currently treated with thrombolytic drugs. Several factors explain this situation: a limited therapeutic window, insufficient public knowledge of the warning signs for stroke, the small number of centers able to administer thrombolysis on a 24-hour basis and an excessive fear of hemorrhagic complications. The aim of this review is to explore the clinical efficacy of treatment with alteplase and consider the hemorrhagic risks.
\end{abstract}

Keywords: rt-PA, acute ischemic stroke, intracranial hemorrhage

\section{Introduction}

Thromboembolic events can cause a wide range of diseases such as ischemic stroke or myocardial infarction. Removal of the obstructing clot was first attempted in myocardial infarction, which remains the only disease commonly treated with thrombolytic drugs. Although recombinant tissue-type plasminogen activator (rt-PA) has been approved for acute ischemic stroke, less than $5 \%$ of qualifying patients actually receive rt-PA. ${ }^{1}$ In addition, rt-PA is the only drug licensed and available for treatment of ischemic stroke that can led to a recanalization of occluded vessels and to an improve in clinical outcome. The aim of this review is to report data on safety and efficacy of rt-PA in treatment of ischemic stroke.

\section{Structure and mechanisms of action}

Tissue plasminogen activator (t-PA) is an endogenous human serine protease found in the intravascular space, in the blood-brain interface, and in the brain parenchyma (neurons, astrocytes, and microglia). ${ }^{2,3}$ t-PA is composed by five conserved domains (finger, epidermal growth factor-like, K1, K2, and catalytic domain) that are differently involved in the pleiotropic functions of the molecule. ${ }^{4}$ t-PA plays a central role in maintaining homeostatic control in the blood coagulation cascade. By cleaving the precursor molecule plasminogen, it produces the active enzyme plasmin, which then dissolves fibrin-based clots in focal cerebral ischemia.

By contrast, in the brain parenchyma, t-PA has been associated with multiple physiologic and pathologic events including synaptic plasticity and cell death. 
In pathologic conditions, t-PA has been linked to neurotoxicity (especially cell injury induced by activation of excitatory amino acid receptors). Like other molecules involved in central nervous system regulation, t-PA is produced by neurons (but also by glial cells), released through exocytocit mechanisms which is antagonized by neuroserpin. ${ }^{5}$ It may thus be classified as a neuromodulator ${ }^{6}$ and its functions may include facilitation of axon elongation (by degradation of the extracellular matrix) and long-term potentiation of memory (LPT). This latter effect seems to be related to potentiation of glutamate (N-methyl-D-aspartic acid [NMDA]) receptor signaling and, more specifically, to the ability to cleave the NR1 subunit of the NMDA receptor, resulting in enhanced $\mathrm{Ca}^{2+}$ influx into the neuron. Moreover, the degradation of the extracellular matrix by t-PA seems to have a role in the physiological effect of t-PA on LTP. ${ }^{7}$

There is considerable release of endogenous t-PA in animal models of stroke, leading to incoordinate effects on NMDA receptor signaling and on the extracellular matrix. Thus, the physiologic effects of t-PA may become deleterious in the setting of cerebral ischemia. ${ }^{7}$ In particular the cleavage of the NR1 subunit of the NMDA receptor seems to play an essential mechanism in NMDA neurotoxicity. ${ }^{8}$

Likewise, destructive effects on the extracellular matrix and the endothelial basal lamina would explain the finding that rt-PA can compromise the integrity of the blood-brain barrier (BBB) and finally cause overt hemorrhage (see below, Hemorrhagic complication). This has been explored at molecular level in animal models $\mathrm{s}^{5,9,10}$ and may be a decisive factor in the risk of symptomatic intracerebral hemorrhage (sICH) in patients treated with rt-PA. ${ }^{11}$ Of course, a central factor for the propensity of rt-PA to cause intracerebral hemorrhage ( $\mathrm{ICH}$ ) may be its ability to cross the BBB by virtue of its proteolytic activity, as observed in animal studies. $^{5}$

\section{Efficacy of intravenous thrombolysis}

In 1995, the National Institute of Neurological Disorders and Stroke (NINDS) study group reported that patients with acute ischemic stroke who received alteplase $(0.9 \mathrm{mg}$ per kilogram of body weight, maximum dose $90 \mathrm{mg}, 10 \%$ given as a bolus with the remainder given over one hour) within three hours after the onset of symptoms were at least $30 \%$ more likely to have minimal or no disability at three months than those who received placebo. ${ }^{12}$ A subsequent study demonstrated that patients treated with rt-PA were also more likely to have minimal or no disability at one-year follow-up. ${ }^{13}$ One year after the publication of the NINDS study, the US Food and Drug Administration approved intravenous rt-PA as a treatment for acute ischemic stroke and this treatment is the only approved medical therapy for patients with acute ischemic stroke to date and is recommended as first-line treatment by most national and international stroke associations. ${ }^{14-16}$

The widespread adoption of treatment with rt-PA has not been without controversy, at least in part because other studies with rt-PA in ischemic stroke have been negative, because of concerns that baseline imbalances in the NINDS report might explain the benefit of this treatment, and because the overall benefit in the trial included a 10-fold increase in the proportion of patients treated with alteplase suffering from $\mathrm{sICH}$ ( $6.4 \%$ versus $0.6 \%$ in the placebo group), which could compromise the benefit when used in routine clinical practice.

Negative thrombolytic studies differed from the NINDS trial in fundamental and important aspects, such as different thrombolytic drugs, different doses of rt-PA, and longer intervals between symptom onset and treatment. Among negative trials of intravenous rt-PA there was the first European Cooperative Acute Stroke Study (ECASS I), in which a higher dose of rt-PA was used and patients were randomized up to six hours after the onset of symptoms. ${ }^{17}$ In the ECASS II trial, the dose of rt-PA was identical to that used in the NINDS trial, but there was a six-hour treatment window, with most patients treated after three hours. In this second trial, the results were not in favor of treatment with alteplase. ${ }^{18}$

The Alteplase Thrombolysis for Acute Non-interventional Therapy in Ischemic Stroke (ATLANTIS) study used a treatment protocol identical to that of the NINDS trial but randomized patients from three to five hours after stroke and also showed negative results. ${ }^{19}$

Despite these findings, Hacke and colleagues reported positive results of an intention-to-treat analysis of pooled data from randomized trials of rt-PA for ischemic stroke (NINDS, ECASS I, ECASS II, and ATLANTIS) that included 2775 patients treated up to six hours after symptom onset in more than 300 hospitals located in 18 countries (Table 1). ${ }^{20}$

This analysis supported the results of the NINDS trial and demonstrated that treatment within three hours (and possibly up to 4.5 hours) of symptom onset is associated with a greater chance of a favorable outcome at three months. This pooled analysis also showed how crucial the time of treatment is; in particular, alteplase is nearly twice as efficacious when administered within the first 1.5 hours after the onset of ischemic stroke as it is when administered within 1.5 to 3 hours after stroke onset (odds ratio for good outcome: 2.81 for an interval of 0 to 90 minutes, 1.55 for 91 to 180 minutes, 
Table I Results of major trials on use of endovenous rt-PA. Report of the 90-day outcome for patients treated with alteplase

\begin{tabular}{lllll}
\hline Name of study & Treatment window & sICH & $\begin{array}{l}\text { Mortality at } \\
\text { three months }\end{array}$ & $\begin{array}{l}\text { Independence } \\
\text { at three months }\end{array}$ \\
\hline NINDS & 3 hours & $10.9 \%$ & $17 \%$ & $47.2 \%$ \\
SITS-MOST & 3 hours & $7.3 \%$ & $11.3 \%$ & $54.8 \%$ \\
ECASS I & 6 hours & $24.9 \%$ & $22.3 \%$ & $41.6 \%$ \\
ECASS II & 6 hours & $8.5 \%$ & $10 \%$ & $40.3 \%$ \\
ATLANTIS & $3-5$ hours & $6.9 \%$ & $17.3 \%$ & $34 \%$ \\
ECASS III & $3-4.5$ hours & $2.4 \%$ & $7.7 \%$ & $52.4 \%$ \\
\hline
\end{tabular}

Abbreviations: ATLANTIS, Alteplase Thrombolysis for Acute Non-interventional Therapy in Ischemic Stroke; ECASS, European Cooperative Acute Stroke Study; NINDS, National Institute of Neurological Disorders and Stroke; rt-PA, tissue plasminogen activator; sICH, symptomatic intracerebral hemorrhage; SITS-MOST, Safe Implementation of Thrombolysis in Stroke-Monitoring Study.

and 1.40 for 181 to 270 minutes). Moreover, the estimated number needed-to-treat (NNT) of patients to identify clinical benefit is only three. ${ }^{21}$

The second concern about alteplase in clinical practice was that the baseline imbalance in stroke severity between the rt-PA- and placebo-treated groups in the NINDS trial might explain the benefit observed in clinical outcome. An independent group reanalyzed the trial data and confirmed a statistically significant treatment benefit despite subgroup imbalances in stroke severity assessed with National Institutes of Health Stroke Survey (NIHSS) score. ${ }^{22}$

Finally, we doubt the possibility that the results reached in the NINDS trial would be generalizable to nonstudy settings. This fear was reinforced by several observational studies that documented higher rates of bleeding complications occurring when treatment protocols were violated. In contrast, other observational studies reported that, in the "real world," results similar to those found in the NINDS trial could be achieved only if treatment protocols were carefully followed. ${ }^{23}$ Moreover, a great number of studies asserted that successful clinical outcomes and low complication rates could be achieved by treating patients with intravenous rt-PA beyond the clinical trial setting. ${ }^{24-27}$ In particular, the Standard Treatment with Alteplase to Reverse Stroke (STARS) study conducted on consecutive acute stroke patients treated with intravenous rt-PA showed results similar to the NINDS study: $13.1 \%$ of patients died within 30 days of follow-up and $11.5 \%$ experienced an $\mathrm{ICH}^{24}$

On the basis of these different results the European Medicines Agency (EMEA) granted approval of alteplase in 2002 under condition that a study be initiated in order to assess the safety and efficacy of rt-PA in routine clinical practice; subsequently, the Safe Implementation of Thrombolysis in Stroke-Monitoring Study (SITS-MOST) was undertaken.
SITS-MOST completed in 2007 and confirmed that alteplase is safe and effective when used in the first three hours after the onset of stroke in a wide range of clinical settings. ${ }^{28}$ The primary aim of the study was to establish whether the levels of safety seen in randomized controlled trial populations could be reproduced in routine clinical practice, and mainly concerned ICH. The results of this observational study showed that the proportion of patients who experience sICH was similar to that observed in randomized controlled trials. Moreover there was a trend towards a reduced incidence of ICH in SITS-MOST when compared to NINDS study, although confidence intervals (CI) overlapped. A reduction in mortality within the first three months was also seen in SITS-MOST compared with randomized controlled trials $(11.3 \%$ vs $17.3 \%$; $95 \% \mathrm{CI}$ $10.5-12.1,14.1-21.1$, respectively). This study was compared with the Canadian Alteplase for Stroke Effectiveness Study (CASES), an observational cohort study on alteplase in ischemic stroke done between February 1999, and June 2001, where mortality was $22.3 \% .^{29}$ Lower mortality in SITS-MOST could be explained with lower stroke severity and lower age in study population in respect to randomized controlled trials and CASES.

Similar results were achieved by smaller observational multicenter studies, including a total of about 650 patients, ${ }^{24,27}$ although one case had a higher rate of sICH. ${ }^{30}$

Moreover the results of SITS-MOST regarding complete recovery at three months follow-up was the same as in the pooled randomized controlled trials ( $39 \%$ vs $42 \%$ CI 36.9-39.7, 38.2-43.2, respectively).

Lyden and colleagues ${ }^{31}$ discussed the uncertainty shown by less experienced centers in including thrombolysis in their routine clinical practice. At the end of SITS-MOST study, the recruitment of "no-expertise" centers had increased to about $50 \%$, thus demonstrating an increase in awareness 
and growth in the ability to provide thrombolysis within the three-hour therapeutic window in clinical practice. Moreover, compared with centers with more experience, sICH was almost the same, which demonstrates that treatment safety could be maintained across all centers. Neither the proportion of patients with sICH nor the degree of independence at three months was significantly different between experienced and new centers. Mortality was a little higher in new centers but remained lower than that seen previously in randomized controlled trials. This data could be explained by higher stroke severity in new centers (one point higher median NIHSS score) than in experienced centers.

It is interesting to note that there has been a gradual decline in the overall initial severity of stroke and in mortality rates among patients enrolled in major randomized studies of acute ischemic stroke over the past two decades. This observation may reflect the trend toward the use of alteplase in patients who have less severe neurologic impairments (as demonstrated by the results of the SITS-MOST trial), as well as the increased number of stroke units in Europe and the improved care provided by such units.

\section{Thrombolysis after three hours}

The second request of the EMEA was the initiation of a third randomized trial, ECASS III, in which the therapeutic window was extended beyond three hours. Before ECASS III, two European trials, the ECASS I and ECASS II, investigated a larger time window (up to six hours) but failed to show efficacy of treatment with alteplase. ${ }^{17,18}$ A subsequent analysis of the NINDS study ${ }^{32}$ and the combined analysis ${ }^{20}$ of data from the six randomized trials, ${ }^{12,17-19,33}$ showed a significant association between efficacy of treatment and the interval between the onset of symptoms and drug administration. Data from the pooled analysis showed a favorable outcome even if treatment was given between 3 and 4.5 hours, with an odds ratio of 1.4 for a good outcome with alteplase treatment as compared with placebo. This analysis also suggested that a longer time window was not associated with higher rates of sICH or with greater mortality. ${ }^{20}$

ECASS III is the second randomized trial (after the NINDS trial in 1995) to show significant treatment efficacy with intravenous alteplase in the unadjusted analysis of the primary end-point demonstrating that patients with acute ischemic stroke benefited from treatment when administered 3 to 4.5 hours after the onset of stroke symptoms. ${ }^{34}$ Moreover this benefit was maintained as significant after adjustment for all prognostic baseline characteristics. In this trial the overall rate of sICH was increased in patient treated with rt-PA as compared with placebo, but mortality was not affected. All these findings are consistent with results from other randomized controlled trials. ${ }^{12,20,35}$

Finally, the initial severity of stroke represented the most predictive factor for the functional and neurologic outcome and for the risk of death. Previous trials failed to show a significant advantage of alteplase therapy in patients who received treatment within 0 to 6 hours after the onset of symptoms. ${ }^{18,19,36}$ It is likely that the time window of up to six hours and the lack of statistical power are responsible for the failure of these studies.

Although it is well known that thrombolysis for ischemic stroke is associated with an increased risk of symptomatic intracranial hemorrhage, it is difficult to compare the incidence of this complication in the various studies due to the different definitions used for intracranial hemorrhage.

The classifications widely used in clinical trials to identify intracranial bleeding are those reported in the NINDS and ECASS studies. ${ }^{12,17,18}$ In the former, hemorrhagic transformations were divided into two radiological groups: hemorrhagic cerebral transformation and intracerebral hematomas. Hemorrhagic cerebral infarction is defined as computed tomography (CT) findings of acute infarction with punctate or variable hypodensity/hyperdensity with an indistinct border within the vascular territory suggested by acute neurologic signs and symptoms. Intracerebral hematoma is defined as a typical homogeneous, hyperdense lesion with a sharp border with or without edema or mass effect within the brain. This hyperdense lesion could arise at a site remote from the vascular territory of the ischemic stroke or within but not necessarily limited to the territory of the presenting ischemic stroke. Also hemorrhage with an intraventricular extension is considered an intracerebral hematoma.

ECASS classification is a purely radiological definition subdivided into four categories: hemorrhagic infarction type 1 (HI-1; small petechiae along the margins of the infarct); hemorrhagic infarction type 2 (HI-2; confluent petechiae within the infracted area, but without space-occupying effect); parenchymal hematoma type 1 (PH-1; an hematoma in less than $30 \%$ of infracted area with some slight spaceoccupying effect); parenchymal hematoma type 2 (PH-2; a dense hematoma in more than $30 \%$ of infarcted area with substantial space-occupying effect, or any hemorrhagic lesion outside the infarcted area).

In the ECASS III trial, sICHs have been identified as the predominant cause of neurologic deterioration. Considering this definition, a difference of $2.14 \%$ was found between the two treatment arms. The authors also analyzed the incidence 
of intracranial hemorrhage using the definition of the other trials ${ }^{12,17,37}$ and in this case the rate of $\mathrm{sICH}$ was no higher than that reported in previous trials or in SITS-MOST, despite the extended time window.

In ECASS III the overall mortality rate was approximately $8 \%$ and was lower than that reported in previous trials and this fact could be also attributable to the inclusion of patient with lower stroke severity (baseline NIHSS score: for ECAS-III, 9; for NINDS, 14).

In comparison with pooled analysis of previous randomized trials, in ECASS III, the odds ratio for a good outcome was 1.34 (CI 181-270 minutes) and the NNT for a good outcome is equal to 14 . The effectiveness of alteplase in an extended time window up to 4.5 hours is confirmed by evaluation of the data from the Safe Implementation of Thrombolysis in Stroke-International Stroke Thrombolysis Register (SITS-ISTR) registry which shows that the rates of sICH, mortality, and independence at three-month followup in routine clinical practice are similar in patients who received treatment between three and 4.5 hours and for those treated within three hours from stroke onset. ${ }^{38}$ In the SITSISTR registry, a small group of patients $(n=664)$ received rt-PA after three hours. In the three to four, five-hour cohort, any hemorrhage occurred in $17 \%$ of patients whereas $8 \%$ had symptomatic intracranial bleeding according to the NINDS definition. Moreover $41 \%$ of patients reached a score of $0-1$ on the modified Rankin score at three months follow-up. The results of both studies (SITS-ISTR and ECASS III) are statistically significant and allow physicians to extend the window of treatment up to 4.5 hours.

\section{Intra-arterial and combined thrombolysis}

Different strategies for treating acute ischemic stroke have included primary intra-arterial (IA) therapy, as reported in several case series and the Prolyse in Acute Cerebral Thromboembolism (PROACT) trials, ${ }^{39-41}$ and combined intravenous/IA therapy. ${ }^{42-47}$

In these studies, neurological improvement was variable with minimal or no neurological deficit reported in $15 \%$ to $75 \%$ of patients. Factors affecting this wide variation in outcome are represented by the system used for assessing outcome, the dose of thrombolytic agent used, the differences in baseline patient characteristics (mainly age and neurological impairment at baseline) and the site of arterial occlusion. In these reports, time from stroke onset is up to six hours with complete recanalization seen in approximately $40 \%$ of patients and partial recanalization in $35 \%{ }^{40,48-51}$ In this report, the rate of recanalization was higher than in other studies with intravenous rt-PA. ${ }^{52}$

The PROACT I and II trials assessed the safety and efficacy of IA thrombolysis. ${ }^{39,40}$ In PROACT I, ${ }^{39}$ stroke patients presenting within six hours of symptom onset with angiographically proven middle cerebral artery (MCA) occlusion were enrolled. Subjects were randomized at prourokinase or placebo over 120 minutes into the proximal thrombus face. Partial or complete recanalization was obtained in $57.7 \%$ of patients treated with prourokinase, whereas hemorrhagic transformation, causing neurologic deterioration within 24 hours, occurred in $15.4 \%$ of cases. However, the number of patients enrolled in this study was too small to reach a statistical significance in clinical outcome, so the PROACT II, a larger, randomized study was started. Also this latter study supported previous reports showing a higher degree of arterial recanalization and a better outcome in patients who underwent IA fibrinolysis compared to placebo group. ${ }^{40}$

All randomized trials were performed in patients with anterior circulation ischemic stroke; this is mainly due to the greater severity and clinical difference of stroke in the vertebrobasilar system. The clinical outcome of patients with vertebrobasilar occlusion seems to be less favorable with higher reported morbidity and mortality, ${ }^{53}$ and reports on the natural history of stroke in posterior circulation shows a global poor outcome with mortality in up to $80 \%$ of cases. ${ }^{54-57}$ In addition, the evolution of clinical signs in posterior circulation stroke differs from those in anterior circulation stroke. In the latter, onset of symptoms is often concomitant with abrupt occlusion of the vessel whereas in the former there often may be a gradual evolution of symptoms. ${ }^{58}$ Patients with vertebrobasilar infarcts are more prone to have severe intracranial large-artery disease which can led to restenosis after thrombolysis. ${ }^{59-61}$

There are only a few reports about intra-arterial thrombolysis in posterior circulation and most extended the time of intervention beyond six hours. ${ }^{61}$ No randomized trials evaluated the safety and efficacy of intra-arterial or endovenous fibrinolysis in vertebrobasilar stroke but, as the natural history of this type of stroke is severe, a neurointerventional approach could be the only lifesaving therapy to show some benefit in patients.

The intravenous administration of rt-PA has the advantage of early treatment initiation, but it is limited by poor recanalization rates with $30 \%$ to $40 \%$ of major occluded arteries that partially or completely recanalize as evaluated by cerebral angiography. ${ }^{52}$ 
The combination of intravenous rt-PA with local IA therapy may improve the chance of achieving a better recanalization rate by taking advantage of both treatments, and could increase clinical recovery as a result of early initiation of therapy and a hypothesized higher recanalization rate; however, this approach may increase the risk of sICH compared with either treatment alone.

The Interventional Management of Stroke (IMS) study ${ }^{42,62}$ was performed using intravenous/IA rt-PA therapy. Patients with an NIHSS score of 10 at baseline had intravenous rt-PA initiated $(0.6 \mathrm{mg} / \mathrm{kg}, 60 \mathrm{mg}$ maximum over 30 minutes $)$ within three hours of onset. Afterwards additional rt-PA was administered intra-arterially at the site of clot up to a total dose of $22 \mathrm{mg}$ over two hours of infusion or until lysis of thrombus was obtained. The analysis of results was made by comparing patients who were treated with placebo or with intravenous rt-PA from the NINDS study. Patients treated with combined intravenous/IA rt-PA had a significant better outcome at three months follow-up compared with placebo arm patients in NINDS trial, while no significant difference was found in the rate of ICH when compared with patients in NINDS treated with rt-PA.

Another pilot study, the Emergency Management of Stroke (EMS) Bridging Trial was performed in order to evaluate safety and efficacy of combined therapy in patients with acute ischemic stroke. ${ }^{43}$ Although the study sample was small, an increased rate of recanalization was observed in the group treated with combined therapy when compared to patients treated with intra-arterial rt-PA alone.

In another report,$^{63}$ despite the highest number of patients with type of vessel occlusion associated with poor prognosis (11 internal carotid artery, and five basilar artery) and a higher baseline NIHSS score in the intravenous/IA versus IA arm, patients in combined therapy showed a more favorable outcome with comparable rates of mortality and numbers of sICH when compared to patients receiving IA therapy alone. There was a trend toward improved clinical outcome in patients receiving intravenous/IA therapy while safety was similar to that of IA therapy alone. The authors attributed this improvement to earlier initiation of treatment, and greater recanalization rates with combined intravenous/IA thrombolysis. Potential disadvantages of combined therapy may include higher total rt-PA doses which could contribute to increases in the rates of cerebral hemorrhagic transformation.

\section{Hemorrhagic complication}

The efficacy of thrombolysis for ischemic stroke has been confirmed since the publication of the NINDS study, but only
$1 \%-8 \%$ of potentially eligible patients are being treated in the United States and in Europe. ${ }^{64}$

Several factors have been reported to explain the underuse of this therapeutic approach to ischemic stroke: the short therapeutic window, insufficient public warning and knowledge of stroke symptoms, the limited number of centers able to perform thrombolysis on a 24-hour basis and an excessive fear of hemorrhagic complications. ${ }^{65}$

Although in clinical practice this complication may be less frequent than failure of treatment to recanalized occluded cerebral artery or early (up to 48 hours) reocclusion, ICH seems to represent an important obstacle to the generalization of thrombolytic therapy.

Intracerebral hemorrhage mostly occurs in the core of the infracted area, thus suggesting that ischemic events can have an important role. ${ }^{37}$ An hemorrhagic transformation can happen spontaneously and sometimes during the early hours of a cerebral infarction. This hemorrhagic risk is clearly increased by administration of an heparin or thrombolytic agent. As reported before, the principal requisition for blood extravasation towards the brain tissue is an alteration of the BBB. ${ }^{11}$ In experimental models of focal cerebral ischemia, the basal lamina of the vessels and the extracellular matrix show an alteration and the adhesion between the microvessel cells and the extracellular matrix is dearranged so there can be an extravasation of blood elements. There is an increase in capillary permeability that comes along with an inrush of plasma components inside the brain tissue, an inflammatory reaction with thrombin activation, and an increasing of many mediators such as platelet-activating factor, tumor necrosis factor $\alpha$ and bradykinin, which contribute to increase endothelial permeability. In addition, oxidative damage may increase hemorrhagic risk. ${ }^{66}$

Matrix metalloproteinases (MMP) are involved in the hemorrhagic transformation, and their activation is partly responsible for the BBB disruption. MMPs represent a family of proteolytic enzymes combined with zinc, which acts normally on the remodeling of the extracellular matrix. Inappropriate activation can induce proteolysis of the matrix of the neurovascular unity (endothelium, astrocyte, and neuron). MMPs are liberated by the endothelium and the polynucleates at the inflammatory stage of ischemia and utilize type IV collagen and laminin as substrates. In some animal models of focal cerebral ischemia, activation of MMP-9 is associated with increased permeability of the BBB that leads to edema formation and hemorrhagic transformation. ${ }^{67-70}$ In some studies, a complementary role of plasmin (which is another serine-protease generated by 
interaction between t-PA and the thrombus) is observed in the disruption of the BBB and the occurrence of $\mathrm{ICH} .{ }^{71}$

MMP-2 and MMP-9 released during the ischemic event can damage the vessel components, particularly type IV collagen, fibronectin, and laminin, thus altering the basal lamina of the cerebral vessels. In humans, elevation of MMP-9 is linked to the severity of ischemic stroke, ${ }^{72}$ and the pretherapeutic MMP-9 rate is an independent predictor of the risk of hemorrhagic transformation related to thrombolysis. ${ }^{73,74}$ The importance of plasma levels of MMP-9 has to be clarified to determine if evaluation of pretreatment MMP-9 levels is likely to improve the benefit-risk ratio of rt-PA administration for stroke in routine use. Moreover the association of a nonspecific inhibitor of MMPs (BB-94) with rt-PA is able to limit the frequency of hemorrhagic transformation without diminishing lytic efficiency. ${ }^{75}$

Also plasma elevation of cellular fibronectin (cFN), a substance synthesized by endothelial cells that reflects microvascular damage, is considered a marker of t-PA-related $\mathrm{ICH}$ probably more specific than MMP-9 concentration. ${ }^{76}$

Preliminary studies have also suggested that there are many other biomarkers that may be able to predict the risk of ICH. Pretreatment levels of endogenous fibrinolysis inhibitors (plasminogen activator inhibitor [PAI-1] and thrombin-activated fibrinolysis inhibitor [TAFI]) could predict rt-PA-related sICH. ${ }^{77}$ Exact knowledge of mechanisms related to $\mathrm{ICH}$ after thrombolysis and the role of biomarkers could be useful in selecting patients that can benefit from such treatment. Other elements must be taken in account for the genesis of rt-PA-related ICH: age, hypertension, diabetes mellitus or cerebral amyloid angiopathy, extent of early ischemic signs shown on brain CT scan or the volume of cerebral ischemic lesions on diffusion weighted MRI, and the presence of leukoaraiosis. ${ }^{17,78-80}$

In the NINDS t-PA Stroke Study, as well as in some meta-analyses, sICH was defined as a CT-documented hemorrhage that was temporally related to any deterioration in the patient's clinical condition. ${ }^{37}$ The post-thrombolysis ICH spectrum ranges from frequent benign hemorrhagic petechiae that can be associated with early reperfusion, to rare large parenchymal hematomas with severe mass effect.

Analysis from the ECASS I and ECASS II studies showed that only the $\mathrm{PH}-2$ type parenchymal hematomas (homogeneous hematomas with mass effect occupying $30 \%$ of ischemic lesion volume) are associated independently with clinical deterioration and poor prognosis. ${ }^{81,82}$

Longer door-to-needle time has been shown to be associated with higher rates of $\mathrm{sICH} .{ }^{83}$ However, the majority of studies have not identified the time to onset of thrombolytic treatment as an independent risk factor of sICH in multivariate analysis, and the pooled analysis of the NINDS, ECASS, and ATLANTIS trials failed to show an independent association between timing of rt-PA administration and risk of cerebral hematoma. ${ }^{20}$

The first trials on rt-PA have provided evidence that higher doses of lytic agents lead to higher rates of $\mathrm{sICH},{ }^{17,84}$ so the dose was limited to $0.9 \mathrm{mg} / \mathrm{kg}$ up to $90 \mathrm{mg}$ in total.

Age has been consistently found to be a risk factor for sICH after thrombolysis for both acute ischemic stroke and myocardial infarction. ${ }^{79,80,86}$ In the ECASS I study, age is the only independent predictive factor of parenchymal hematomas, with an odds ratio of 1.3 for every 10 year increase in age ${ }^{17}$ Higher hemorrhagic risk could be linked to high frequency of microangiopathic processes, especially cerebral amyloid angiopathy in elderly patients and to the presence of leucoaraiosis. ${ }^{87}$ In the study by Heuschmann and colleagues, the rate of sICH increased with age from $4.9 \%$ in patients younger than 55 years to $10.3 \%$ in patients aged 75 years and older. ${ }^{88}$ In some European countries, rt-PA is not recommended as treatment of ischemic stroke in patients older than 80 years. Recent data from several open-label studies on use of rt-PA have shown that the risk of sICH in the elderly is comparable to that of younger patients. ${ }^{89-91}$ In particular the CASES trial showed that the benefit-risk ratio of intravenous rt-PA can be favorable in carefully selected elderly stroke patients treated within three hours. ${ }^{91}$ The sICH rate was $4.4 \%$ in the group of patients aged 80 years or older included in this study. The Stroke Survey Group rt-PA analysis also concluded that it was not justified to systematically contraindicate thrombolysis for patients older than 80 years. ${ }^{89}$

Many authors have shown the importance of the baseline stroke severity in hemorrhagic risk after thrombolysis. ${ }^{12,78,85,92}$ In the NINDS study, patients with a baseline NIHSS score of 20 were 11 times more prone to having sICH than patients with an NIHSS score of $5 .{ }^{12}$ The Multicentre tPA Acute Stroke Survey study also showed that the NIHSS score was an independent marker of $\mathrm{ICH}$, with an odds ratio of 1.38 for a one-point increase in the NIHSS score. ${ }^{89}$ On the basis of these results, in some countries a NIHSS score of 25 represents a contraindication for alteplase administration. In contrast to the NINDS study, Sylaja and colleagues on their report did not find a significant association of severity of neurological deficit at baseline with increased risk of sICH. ${ }^{91}$ Moreover, the ECASS I trial showed that severity of neurological deficit at admission represented a risk factor 
for hemorrhagic transformation and not for parenchymal hematoma. ${ }^{79}$

Another factor which may contribute to the development of rt-PA-related sICH is hypertension during the first 24 hours after ischemic stroke, ${ }^{78}$ this data underlies the importance of close surveillance of blood pressure during the first 24 hours and the benefit of applying a strict therapeutic protocol for the control of hypertension, similar to the one used by the NINDS study, to limit hemorrhagic risk.

It is well known that patients with hyperglycemia have worse outcomes when compared to those with normal blood glucose on presentation. ${ }^{93}$ Experimental and human studies indicate that hyperglycemia predicts higher stroke mortality independently from stroke severity, stroke type, or age. These data suggest that hyperglycemia may directly contribute to poor outcomes by exacerbating acute brain injury. ${ }^{94-96}$ Alternatively, it has been proposed that hyperglycemia is simply a bystander phenomenon, a catecholamine-based stress response to a more severe stroke, which may not have any direct causative role in the poor prognosis that is observed among these patients. ${ }^{97-99}$

Moreover several studies have shown the influence of baseline glycemia on the risk of ICH after thrombolysis. ${ }^{85,100,101}$ In a study published in 1999, patients with a glycemia value of $\geq 200 \mathrm{mg} / \mathrm{dl}$ on admission showed a symptomatic hematoma rate of $25 \%$ after treatment by t-PA. ${ }^{100}$ In the PROACT II study, there was an increased risk of sICH in patients with pretherapeutic glycemia higher than $200 \mathrm{mg} / \mathrm{dl} .^{102}$

The mechanism of hyperglycemia-related $\mathrm{ICH}$ is not clear. There are numerous animal experimental proofs that hyperglycemia provokes microvascular lesions as well as BBB damage, leading to hemorrhagic transformation of the cerebral infarction. ${ }^{103}$ Moreover Sylaja and colleagues ${ }^{91}$ did not find that a history of diabetes mellitus was a risk factor for sICH, despite the fact that many patients with diabetes mellitus had elevated serum glucose at stroke onset. This may be due to the fact that it is the baseline glucose level, not diabetes mellitus per se, which is predictive of ICH. ${ }^{100}$

The deleterious effect of rt-PA attributed to MMPs worsened in the presence of hyperglycemia and a glycemia value above $400 \mathrm{mg} / \mathrm{dl}$ represents a contraindication to cerebral thrombolysis by intravenous t-PA.

The significance of early ischemic changes on baseline brain CT scan as predictors of hemorrhagic transformation scan remains controversial. ${ }^{104,105}$ The ECASS I and ECASS II trials have shown that the presence of early ischemic change in more than one-third of the MCA territory before thrombolysis is accompanied by an increase in the hemorrhagic transformation risk and poor clinical outcome. In a paper by Tanne and colleagues, the symptomatic ICH rate is multiplied by more than four in patients with such signs on CT. ${ }^{85}$ In contrast, other studies on thrombolysis have shown that the presence of ischemic change in CT scan is related to stroke severity but is not independently associated with the occurrence of ICH. ${ }^{106,107}$

One concern regards the sensitivity and reproducibility of early ischemic change detection that depend on the quality of the CT scanner used and on the experience of the reader. Interobserver reproducibility can be improved by a standardized reading of the CT scan according to the Alberta Stroke Program Early CT Score (ASPECTS) score. ${ }^{107}$

A recent retrospective multicenter study evaluated whether leukoaraiosis is a risk factor for sICH in patients treated with alteplase (endovenous or IA) for anterior circulation stroke. ${ }^{108}$ All patients had received magnetic resonance imaging (MRI) evaluation before thrombolysis and for statistical analysis. Leukoaraiosis in the deep white matter was dichotomized into absent or mild versus moderate or severe. The rate of sICH was significantly higher in patients with moderate to severe leukoaraiosis than in patients without relevant leukoaraiosis (10.5\% vs $3.8 \%$; $\mathrm{p}<0.005$ ), with an odds ratio of 2.9. In the logistic regression analysis (including age, NIHSS score on admission, and type of thrombolytic treatment), leukoaraiosis remained an independent risk factor.

The risk of $\mathrm{ICH}$ after thrombolysis in ischemic stroke patients carrying old asymptomatic microbleeds (which can considered as a marker of microangiopathy, and of amyloid angiopathy remains a controversial subject. ${ }^{109-112}$ Only few studies have performed pretherapeutic T2* MRI sequences to evaluate the presence of microbleeds and their influence on $\mathrm{ICH}$ risk.

Two case series ${ }^{112,113}$ reported the same (18\% and $16 \%$, respectively) incidence of some microbleeds on the pretherapeutic T2* MRI sequence. In both cases none of the patients treated with alteplase experienced sICH. Moreover, in a recently published pooled analysis of 570 patients, the presence of microbleeds (detected in 86 patients) was not predictive of sICH after thrombolysis. ${ }^{114}$ This limited data does not support the exclusion from thrombolysis of stroke patients carrying a few microbleeds on pretherapeutic T2* sequences.

Some authors have suggested that the differences between symptomatic and asymptomatic ICHs are due to the intensity of bleeding rather than physiopathologic 
differences. For others, hemorrhagic infarctions and parenchymal hematomas after t-PA have a different clinical, etiologic, and biological significance. ${ }^{115}$ Benign hemorrhagic transformation can be associated with the natural history of ischemic stroke while parenchymal hematomas, especially the PH-2 type, could be linked to the t-PA itself and particularly to its impact on homeostasis (as demonstrated by elevation of fibrin degradation products [FDP] after treatment). ${ }^{116}$

Adherence to the inclusion criteria of the NINDS trial represents a key point for a favorable benefit-risk ratio in the routinely administration of alteplase for ischemic stroke.

However, these inclusion criteria are more than 10 years old and can appear obsolete as they do not take into account the development of new diagnostic tools. In particular the development of new MRI sequences and the identification of the new biomarkers (MMP-9, PAI-1, TAFI, and cFN) can be useful in assess the benefit-risk ratio.

A recent multicenter study compared the clinical evolution of patients selected on the basis of MRI and treated by intravenous t-PA within the first six hours to that of patients included in the ATLANTIS, ECASS, and NINDS studies. ${ }^{117}$ Patients without significant perfusion-diffusion mismatch and patients with an extended lesion on the diffusion sequence ( $50 \%$ of the MCA territory) were not treated with alteplase. In the group of patients selected on the basis of MRI, the rate of sICH was significantly lower (3\% vs $8 \% ; p=0.01)$ and a favorable outcome could be achieved in $48 \%$.

The same results were obtained in a recent study which evaluated the efficacy of thrombolysis treatment within and beyond the three-hour time window when the decision was based on MRI versus CT scan. ${ }^{118}$ Also in this report, even if treated after the three-hour window, when selected on the basis of MRI results, patients had a significantly lower risk than for $\mathrm{sICH}(3 \%$ vs $9 \% ; \mathrm{p}=0.013)$ and lower mortality (12\% vs $21 \%$; $=0.021)$ (Table 2 ).

\section{Conclusion}

The introduction of rt-PA in clinical practice for the treatment of acute ischemic stroke has represented an important step in the therapeutic approach to a difficult and once untreatable clinical condition. Its major complication, ICH, describes complex and heterogeneous groups of phenomenon, and involves multiple demographic, clinical, biological, and hemodynamic parameters, knowledge of which remains partial. Identification of the risk factors for sICH will help in improved selection of patients for thrombolysis.

The future of t-PA for the treatment of patients with acute ischemic stroke largely depends on successfully attaining several goals including: (i) a better organization of the health care system, (ii) improvement of the thrombolytic properties of t-PA, (iii) inhibition of the deleterious effects of t-PA on the permeability of the neurovascular unit without interfering with its thrombolytic properties, (iv) attenuation of the neurotoxic effects of t-PA, and (v) extension of the time window. The organization of the health care system should include educational programs directed to the public and the development of dedicated acute stroke units staffed by personnel with expertise in the diagnosis and treatment of acute stroke.

The improvement of the efficiency of t-PA as a thrombolytic agent may be based on the results of ongoing studies which are testing the effect of combined intravenous and intra-arterial thrombolysis in addition to the simultaneous use of t-PA and transcranial or local procedures; among these is the EKOS 1-catheter-delivered ultrasound trial by EKOS Corporation. ${ }^{119}$

Moreover, because the effect of t-PA on the BBB permeability seems to be have an important role in the deleterious effects of t-PA itself in the ischemic brain, the

Table 2 Risk factors for hemorrhagic transformation in acute ischemic stroke following thrombolysis

\begin{tabular}{lll}
\hline Clinical factors & Biochemical markers & Radiologic factors \\
\hline $\begin{array}{l}\text { Thrombolytic agent (dose, route of administration, } \\
\text { time of onset to thrombolysis, type of agent) }\end{array}$ & Elevated blood glucose & Early ischemic change on CT \\
Stroke severity & Elevated red cell count & $\begin{array}{l}\text { Large volume infarct, edema, or mass effect } \\
\text { on the baseline CT }\end{array}$ \\
Advancing age & Elevated matrix metalloproteinase & Presence of lacune \\
History of diabetes & Elevated calcium-binding proteins & Reduced blood volume on PWI \\
Elevated blood pressure & Early fibrinogen degradation coagulopathy & Breakdown of blood-brain barrier \\
History of cardiac failure & & Presence of microbleeds on MRI \\
& & Leukoariosis on MRI
\end{tabular}

Abbreviations: $\mathrm{CT}$, computed tomography; MRI, magnetic resonance imaging; PWI, perfusion-weighted image. 
combined therapy of t-PA and protectors of the integrity of the neurovascular unit might achieve promising results. The targets of neuroprotective experiments have included calcium antagonists, sodium channel blockade, glutamate/NMDA antagonists, free radical scavengers, apoptosis inhibitors, $\gamma$-aminobutyric acid-A (GABAA) agonists, and agents that stabilize the neuronal membrane. Unfortunately almost all the trials conducted with such neuroprotective agents failed to shown a substantial benefit in ischemic stroke patients.

Based on the observation that the interaction of t-PA with the NMDA receptor occurs through a particular kringle domain, ${ }^{120}$ new t-PA muteins lacking this domain or containing mutations were developed with the aim to minimize the NMDA-receptor-dependent neurotoxic effects of alteplase to be less harmful when administered in ischemic stroke patients.

\section{Disclosure}

The authors report no conflicts of interest in this work.

\section{References}

1. Molina CA, Saver JL. Extending reperfusion therapy for acute ischemic stroke: emerging pharmacological, mechanical, and imaging strategies. Stroke. 2005;36:2311-2320.

2. Siao CJ, Fernandez SR, Tsirka SE. Cell type-specific roles for tissue plasminogen activator released by neurons or microglia after excitotoxic injury. J Neurosci. 2003;23:3224-3242.

3. Nicole O, Docagne F, Ali C, et al. The proteolytic activity of tissueplasminogen activator enhances NMDA receptor-mediated signaling. Nat Med. 2001;7:59-64.

4. Yepes M, Roussel BD, Ali C, Vivien D. Tissue-type plasminogen activator in the ischemic brain: more than a thrombolytic. Trends Neurosci. 2009;32(1):48-55.

5. Yepes M, Lawrence DA. Tissue-type plasminogen ayctivator and neuroserpin: A well-balanced act in the nervous system? Cardiovasc Med. 2004;14:173-180.

6. Fernandez-Monreal M, Lopez-Atalaya JP, Benchenane K, et al. Is tissue-type plasminogen activator a neuromodulator? Mol Cell Neurosci. 2004;25:594-601.

7. Benchenane K, Lopez-Atalaya JP, Fernandez-Monreal M, Touzani O, Vivien D. Equivocal roles of tissue-type plasminogen activator in stroke-induced injury. Trends Neurosci. 2004;27:155-160.

8. Castel H, Roussel BD, Ali C, et al. Tissue plasminogen activator (tPA)dependent cleavage of the NMDA receptor NR1 subunit occurs in vivo: Relevance to stroke. Atlanta, GA: Proceedings 36th Annual Meeting of the Society for Neuroscience, October 14-18, 2006.

9. Sumii T, Lo EH. Involvement of matrix metalloproteinase in thrombolysis-associated hemorrhagic transformation after embolic focal ischemia in rats. Stroke. 2002;33:831-836.

10. Pfefferkorn T, Wiessner C, Allegrini PR, Kloss CUA, Hamann GF. MRI predicts area of increased plasminogen activation in permanent focal cerebral ischemia. Neurol Res. 2002;24:822-824.

11. del Zoppo GJ, von Kummer R, Hamann GF. Ischemic damage of brain microvessels: inherent risks for thrombolytic treatment in stroke. J Neurol Neurosurg Psychiatry. 1998;65:1-9.

12. Tissue plasminogen activator for acute ischemic stroke. The National Institute of Neurological Disorders and Stroke rt-PA Stroke Study Group. N Engl J Med. 1995;333:1581-1587.
13. Kwiatkowski TG, Libman RB, Frankel M, Tilley BC, Morgenstern LB, $\mathrm{Lu} \mathrm{M}$, et al. Effects of tissue plasminogen activator for acute ischemic stroke at one year. N Engl J Med. 1999;340:1781-1787.

14. Karolinska Stroke Update. Consensus statement on thrombolysis. Nov. 13-14, 2006. Cited on Apr 16, 2009. Available from: http://www. strokeupdate.org/ALLCURRENT/Consensus/Consensus_2000/ 2000.htm.

15. Adams HP Jr, Adams RJ, Brott T, et al. Guidelines for the early management of patients with ischemic stroke: a scientific statement from the Stroke Council of the American Stroke Association. Stroke. 2003;34:1056-1083.

16. The European Stroke Initiative Executive Committee and European Stroke Initiative Writing Group. European stroke initiative recommendations for stroke management — update 2003. Cerebrovasc Dis. 2003;16:311-337.

17. Hacke W, Kaste M, Fieschi C, et al. Intravenous thrombolysis with recombinant tissue plasminogen activator for acute hemispheric stroke. JAMA. 1995;274:1017-1025.

18. Hacke W, Kaste M, Fieschi C, et al. Randomised double-blind placebo-controlled trial of thrombolytic therapy with intravenous alteplase in acute ischaemic stroke (ECASS II). Second EuropeanAustralasian Acute Stroke Study Investigators. Lancet. 1998;352: 1245-1251.

19. Clark WM, Wissman S, Albers GW, Jhamandas JH, Madden KP, Hamilton S. Recombinant tissue-type plasminogen activator (alteplase) for ischemic stroke 3 to 5 hours after symptom onset: the ATLANTIS study: a randomized controlled trial. JAMA. 1999;282:2019-2026.

20. Hacke W, Donnan G, Fieschi C, et al. Association of outcome with early stroke treatment: pooled analysis of ATLANTIS, ECASS, and NINDS rt-PA stroke trials. Lancet. 2004;363:768-774.

21. Saver JL. Number needed to treat estimates incorporating effects over the whole range of clinical outcomes: novel derivation method and application to thrombolytic therapy for acute stroke. Arch Neurol. 2004;61:1066-1070.

22. Ingall TJ, O'Fallon WM, Asplund K, et al. Findings from the reanalysis of the NINDS Tissue Plasminogen Activator for Acute Ischemic Stroke Treatment Trial. Stroke. 2004;35:2418-2424.

23. Wardlaw JM, Zoppo G, Yamaguchi T, Berge E. Thrombolysis for acute ischaemic stroke. Cochrane Database Syst Rev. 2003;3:CD000213.

24. Wang DZ, Rose JA, Honings DS, Garwacki DJ, Milbrandt JC. Treating acute stroke patients with intravenous tPA: the OSF stroke network experience. Stroke. 2000;31:77-81.

25. Albers GW, Bates VE, Clark WM, Bell R, Verro P, Hamilton SA. Intravenous tissue-type plasminogen activator for treatment of acute stroke: the Standard Treatment with Alteplase to Reverse Stroke (STARS) study. JAMA. 2000;283:1145-1150.

26. Chiu D, Krieger K, Villar-Cordova C, et al. Intravenous tissue plasminogen activator for acute ischemic stroke: feasibility, safety and efficacy in the first year of clinical practice. Stroke. 1998;29:18-22.

27. Grond M, Stenzel C, Schmulling S, et al. Early intravenous thrombolysis for acute ischemic stroke in a community-based approach. Stroke. 1998;29:1544-1549.

28. Tanne D, Bates VE, Verro P, et al. Initial clinical experience with IV tissue plasminogen activator for acute ischemic stroke: a multicenter study. Neurology. 1999;53:424-427.

29. Wahlgren N, Ahmed N, Dávalos A, et al. Thrombolysis with alteplase for acute ischemic stroke in the Safe Implementation of Thrombolysis in Stroke-Monitoring Study (SITS-MOST): an observational study. Lancet. 2007;369:275-282.

30. Hill MD, Buchan AM. Thrombolysis for acute ischemic stroke: results of the Canadian Alteplase for Stroke Effectiveness Study. CMAJ. 2005;172:1307-1312.

31. Katzan IL, Furlan AJ, Lloyd LE, et al. Use of tissue-type plasminogen activator for acute ischemic stroke: the Cleveland area experience. JAMA. 2000;283:1151-1158.

32. Lyden PD, Lees KR, Davis SM. Alteplase for acute stroke revisited: the first 10 years. Lancet Neurol. 2006;5:722-724. 
33. Marler JR, Tilley BC, Lu M, et al. Early stroke treatment associated with better outcome: the NINDS rt-PA Stroke Study. Neurology. 2000;55:1649-1655.

34. Clark WM, Albers GW, Madden KP, Hamilton S. The rt-PA (alteplase) 0- to 6-hour acute stroke trial, part A (A0276g): results of a double-blind, placebo-controlled, multicenter study. Stroke. 2000;31: 811-816.

35. Hacke W, Kaste M, Bluhmki E, et al; ECASS Investigators. Thrombolysis with alteplase 3 to 4.5 hours after acute ischemic stroke. $N E n g l$ J Med. 2008;359(13):1317-1329.

36. Lansberg MG, Albers GW, Wijman CA. Symptomatic intracerebral hemorrhage following thrombolytic therapy for acute ischemic stroke: a review of the risk factors. Cerebrovasc Dis. 2007;24:1-10.

37. Savitz SI, Lew R, Bluhmki E, Hacke W, Fisher M. Shift analysis versus dichotomization of the modified Rankin scale outcome scores in the NINDS and ECASS II trials. Stroke. 2007;38:3205-3212.

38. Intracerebral hemorrhage after intravenous t-PA therapy for ischemic stroke. The NINDS t-PA Stroke Study Group. Stroke. 1997;28: 2109-2118.

39. Wahlgren N, Ahmed N, Dávalos A, et al; SITS investigators. Thrombolysis with alteplase $3-4.5 \mathrm{~h}$ after acute ischemic stroke (SITS-ISTR): an observational study. Lancet. 2008;372(9646):1303-1309.

40. del Zoppo GJ, Higashida RT, Furlan AJ, Pessin MS, Rowley HA, Gent M. PROACT: A phase II randomized trial of recombinant prourokinase by direct arterial delivery in acute middle cerebral artery stroke; PROACT investigators, prolyse in acute cerebral thromboembolism. Stroke. 1998;29:4-11.

41. Furlan A, Higashida R,Weschler L, et al. Intra-arterial prourokinase for acute ischemic stroke. The PROACT II study: a randomized controlled trial. Prolyse in Acute Cerebral Thromboembolism. JAMA. 1999;282:2003-2011.

42. Suarez JI, Sunshine JL, Tarr R, et al. Predictors of clinical improvement, angiographic recanalization, and intracranial hemorrhage after intra-arterial thrombolysis for acute ischemic stroke. Stroke. 1999; 30:2094-2100.

43. The IMS Study Investigators. Combined intravenous and intra-arterial recanalization for acute ischemic stroke: The interventional management of stroke study. Stroke. 2004;35:904-912.

44. Lewandowski C, Frankel M, Tomsick T, et al. Combined intravenous and intra-arterial r-TPA versus intra-arterial therapy of acute ischemic stroke: Emergency Management of Stroke (EMS) Bridging Trial. Stroke. 1999;30:2598-2605.

45. Zaidat OO, Welter E, Love TE, et al. Combined intravenous and intraarterial versus intra-arterial recombinant tissue plasminogen activator in acute ischemic stroke patients: Propensity score analysis [abstract]. J Neurosurg. 2004;100:A181.

46. Ernst R, Pancioli A, Tomsick T, et al. Combined intravenous and intraarterial recombinant tissue plasminogen activator in acute ischemic stroke. Stroke. 2000;31:2552-2557.

47. Zaidat OO, Suarez JI, Santillan C, et al. Intra-arterial and combined intravenous and intra-arterial thrombolytic therapy for distal carotid artery occlusion. Stroke. 2002;33:1828-1833.

48. Suarez JI, Zaidat OO, Sunshine JL, Tarr R, Selman WR, Landis DM Endovascular administration after intravenous infusion of thrombolytic agents for the treatment of patients with acute ischemic strokes. Neurosurgery. 2002;50:251-260.

49. Jansen O, von Kummer R, Forsting M. Thrombolytic therapy in acute occlusion of the intracranial internal carotid artery bifurcation. AJNR Am J Neuroradiol. 1995;16:1977-1986.

50. Theron J, Courtheoux P, Casasco A, et al. Local intraarterial fibrinolysis in the carotid territory. AJNR Am J Neuroradiol. 1989;10:753-765.

51. Ueda T, Sakaki S, Kumon Y, Ohta S. Multivariable analysis of predictive factors related to outcome at 6 months after intra-arterial thrombolysis for acute ischemic stroke. Stroke. 1999;30:2360-2365.

52. Jahan R, Duckwiler GR, Kidwell CS, et al. Intraarterial thrombolysis for treatment of acute stroke: experience in 26 patients with long-term follow-up. AJNR Am J Neuroradiol. 1999;20:1291-1299.
53. Wolpert SM, Bruckmann H, Greenlee R, Wechsler L, Pessin MS, del Zoppo GJ. Neuroradiologic evaluation of patients with acute stroke treated with recombinant tissue plasminogen activator. The rt-PA Acute Stroke Study Group. AJNR Am J Neuroradiol. 1993;14:3-13.

54. Bruckmann H, Ferbert A, del Zoppo GJ, et al. Acute basilar thrombosis: angiologic-clinical comparison and therapeutic implications. Acta Radiol Suppl. 1986;369:38-42.

55. Archer CR, Horenstein S. Basilar artery occlusion: clinical and radiological correlation. Stroke. 1977;8:383-390.

56. Zeumer H, Freitag HJ, Grzyska U, Neunzig HP. Local intraarterial fibrinolysis in acute vertebrobasilar occlusion. Technical developments and recent results. Neuroradiology. 1989;31:336-340.

57. Rosa A, Gautier J. Thrombolytic agents in cerebral infarctions. Rev Neurol. 1991;47:99-110.

58. Kubic C, Adams R. Occlusion of the basilar artery: a clinical and pathological study. Brain. 1946;69:73-121.

59. Ferbert A, Bruckmann H, Drummen R. Clinical features of proven basilar artery occlusion. Stroke. 1990;21:1135-1142.

60. Bogousslavsky J, Regli F, Maeder P, Meuli R, Nader J. The etiology of posterior circulation infarcts: a prospective study using magnetic resonance imaging and magnetic resonance angiography. Neurology. 1993;43:1528-1533.

61. Hacke W, Zeumer H, Ferbert A, Brückmann H, del Zoppo GJ. Intraarterial thrombolytic therapy improves outcome in patients with acute vertebrobasilar disease. Stroke. 1988;19:1216-1222.

62. Becker KJ, Monsein LH, Ulatowski J, Mirski M, Williams M, Hanley DF. Intraarterial thrombolysis in vertebrobasilar occlusion. AJNR Am J Neuroradiol. 1996;17:255-262.

63. IMS II Trial Investigators. The Interventional Management of Stroke (IMS) II Study. Stroke. 2007;38:2127-2135.

64. Wolfe T, Suarez JI, Tarr RW, et al. Comparison of combined venous and arterial thrombolysis with primary arterial therapy using recombinant tissue plasminogen activator in acute ischemic stroke. $J$ Stroke Cerebrovasc Dis. 2008;17(3):121-128.

65. Reeves MJ, Arora S, Broderick JP, et al. Acute stroke care in the US: results from the 4 pilot prototypes of the Paul Coverdell National Acute Stroke Registry. Stroke. 2005;36:1232-1240.

66. Caplan LR. Stroke thrombolysis: slow progress. Circulation. 2006; 114:187-190.

67. del Zoppo GJ, Zeumer H, Harker LA. Thrombolytic therapy in stroke: possibilities and hazards. Stroke. 1986;7:595-607.

68. Rosenberg GA, Estrada EY, Dencoff JE. Matrix metalloproteinases and TIMPS are associated with blood brain barrier opening after reperfusion in rat brain. Stroke. 1998;29:2189-2195.

69. Asahi M, Wang X, Mori T, et al. Effects of matrix metalloproteinase-9 gene knockout on the proteolysis of blood-brain barrier and white matter components after cerebral ischemia. J Neurosci. 2001;21:7724-7732.

70. Sumii T, Lo EH. Involvement of matrix metalloproteinase in thrombolysis-associated hemorrhagic transformation after embolic focal ischemia in rats. Stroke. 2002;33:831-836.

71. Romanic AM, White RF, Arleth AJ, Ohlstein EH, Barone FC. Matrix metalloproteinase expression increases after cerebral focal ischemia in rats. Inhibition of matrix metalloproteinase-9 reduces infarct size. Stroke. 1998;29:1020-1030.

72. Gautier S, Petrault O, Gele P, et al. Involvement of thrombolysis in recombinant tissue plasminogen activator-induced cerebral hemorrhages and effect on infarct volume and postischemic endothelial function. Stroke. 2003;34:2975-2979.

73. Montaner J, Alvarez-Sabin J, Molina C, et al. Matrix metalloproteinase expression after human cardioembolic stroke: temporal profile and relation to neurological impairment. Stroke. 2001;32:1759-1766.

74. Montaner J, Molina CA, Monasterio J, et al. Matrix metalloproteinase-9 pretreatment level predicts intracranial hemorrhagic complications after thrombolysis in human stroke. Circulation. 2003;107:598-603.

75. Castellanos M, Leira R, Serena J, et al. Plasma metalloproteinase-9 concentration predicts hemorrhagic transformation in acute ischemic stroke. Stroke. 2003;34:40-46. 
76. Lapchak PA, Chapman DF, Zivin JA. Metalloproteinase inhibition reduces thrombolytic (tissue plasminogen activator)-induced hemorrhage after thromboembolic stroke. Stroke. 2000;31:3034-3040.

77. Castellanos M, Leira R, Serena J, et al. Plasma cellular-fibronectin concentration predicts hemorrhagic transformation after thrombolytic therapy in acute ischemic stroke. Stroke. 2004;35:1671-1676.

78. Ribo M, Montaner J, Molina CA, et al. Admission fibrinolytic profile is associated with symptomatic hemorrhagic transformation in stroke patients treated with tissue plasminogen activator. Stroke. 2004;35: 2123-2127.

79. Derex L, Hermier M, Adeleine P, et al. Clinical and imaging predictors of intracerebral hemorrhage in stroke patients treated with intravenous tissue plasminogen activator. J Neurol Neurosurg Psychiatry. 2005;76:70-75.

80. Larrue V, von Kummer R, del Zoppo G, Bluhmki E. Hemorrhagic transformation in acute ischemic stroke. Potential contributing factors in the European Cooperative Acute Stroke Study. Stroke. 1997;28: 957-960.

81. Larrue V, von Kummer R, Müller A, Bluhmki E. Risk factors for severe hemorrhagic transformation in ischemic stroke patients treated with recombinant tissue plasminogen activator. A secondary analysis of the European-Australasian Acute Stroke Study (ECASS II). Stroke. 2001;32:438-441.

82. Berger C, Fiorelli M, Steiner T, et al. Hemorrhagic transformation of ischemic brain tissue. Asymptomatic or symptomatic? Stroke. 2001; 32:1330-1335.

83. Fiorelli M, Bastianello S, von Kummer R, et al. Hemorrhagic transformation within 36 hours of a cerebral infarct: relationships with early clinical deterioration and 3-month outcome in the European Cooperative Acute Stroke Study I (ECASS I) cohort. Stroke. 1999;30: 2280-2284

84. Cocho D, Borrell M, Marti-Fabregas J, et al. Pretreatment hemostatic markers of symptomatic intracerebral hemorrhage in patients treated with tissue plasminogen activator. Stroke. 2006;37:996-999.

85. Levy D, Brott T, Haley EC Jr, et al. Factors related to intracranial hematoma formation in patients receiving tissue-type plasminogen activator for acute ischemic stroke. Stroke. 1994;25:291-297.

86. Tanne D, Kasner SE, Demchuk AM, et al. Markers of increased risk of intracerebral hemorrhage after intravenous recombinant tissue plasminogen activator for acute ischemic stroke in clinical practice: the Multicenter rt-PA Acute Stroke Survey. Circulation. 2002;105: $1679-1685$.

87. Gurwitz JH, Gore JM, Goldberg RJ, et al. Risk for intracranial hemorrhage after tissue plasminogen activator treatment for acute myocardial infarction. Participants in the National Registry of Myocardial Infarction 2. Ann Intern Med. 1998;129:597-604.

88. Itoh Y, Yamada M, Hayakawa M, Otomo E, Miyatake T. Cerebral amyloid angiopathy: a significant cause of cerebellar as well as lobar hemorrhage in the elderly. J Neurol Sci. 1993;116:135-141.

89. Heuschmann PU, Kolominsky-Rabas PL, Roether J, et al. Predictors of in-hospital mortality in patients with acute ischemic stroke treated with thrombolytic therapy. JAMA. 2004;292:1831-1838.

90. Tanne D, Gorman MJ, Bates VE, et al. Intravenous tissue plasminogen activator for acute ischemic stroke in patients aged 80 years and older: the tPA stroke survey experience. Stroke. 2000;31:370-375.

91. Berrouschot J, Röther J, Glahn J, Kucinski T, Fiehler J, Thomalla G. Outcome and severe hemorrhagic complications of intravenous thrombolysis with tissue plasminogen activator in very old ( $>$ or $=80$ years) stroke patients. Stroke. 2005;36:2421-2425.

92. Sylaja PN, Cote R, Buchan AM, Hill MD; Canadian Alteplase for Stroke Effectiveness Study (CASES) Investigators. Thrombolysis in patients older than 80 years with acute ischaemic stroke: Canadian Alteplase for Stroke Effectiveness Study. J Neurol Neurosurg Psychiatry. 2006;77:826-829.

93. Wardlaw JM, Dorman PJ, Caldelise L, Signorini DF. The influence of baseline prognostic variables on outcome after thrombolysis. MASTItaly Collaborative group. J Neurol 1999;246:1059-1062
94. Capes SE, Hunt D, Malmberg K, Pathak P, Gerstein HC. Stress hyperglycemia and prognosis of stroke in nondiabetic and diabetic patients: a systematic overview. Stroke. 2001;32:2426-2432.

95. Weir CJ, Murray GD, Dyker AG, Lees KR. Is hyperglycaemia an independent predictor of poor outcome after acute stroke? Results of a long-term follow up study. BMJ. 1997;314:1303-1306.

96. Jorgensen H, Nakayama H, Raaschou HO, Olsen TS. Stroke in patients with diabetes. The Copenhagen Stroke Study. Stroke. 1994;25: 1977-1984.

97. van Kooten F, Hoogerbruge N, Naarding P, Koudstaal PJ. Hyperglycemia in the acute phase of stroke is not caused by stress. Stroke. 1993;24:1129-1132.

98. Murros K, Fogelholm R, Kettunen S, Vuorela AL, Valve J. Blood glucose, glycosylated haemoglobin, and outcome of ischemic brain infarction. J Neurol Sci. 1992;111:59-64.

99. Murros K, Fogelholmd R. Diabetes and stress hyperglycaemia in stroke. J Neurol Neurosurg Psychiatry. 1993;56:428.

100. Woo E, Ma JT, Robinson JD, Yu YL. Hyperglycemia is a stress response in acute stroke. Stroke. 1988;19:1359-1364.

101. Demchuk AM, Morgenstern LB, Krieger DW, et al. Serum glucose level and diabetes predict tissue plasminogen activator-related intracerebral hemorrhage in acute ischemic stroke. Stroke. 1999;30:34-39.

102. Lindsberg PJ, Soinne L, Roine RO, et al. Community-based thrombolytic therapy of acute ischemic stroke in Helsinki. Stroke. 2003;34: 1443-1449.

103. Kase CS, Furlan AJ, Wechsler LR, et al. Cerebral hemorrhage after intra-arterial thrombolysis for ischemic stroke: the PROACT II trial. Neurology. 2001;57(9):1603-1610.

104. Hamann GF, del Zoppo GJ, von Kummer R. Hemorrhagic transformation of cerebral infarction: possible mechanisms. Thromb Haemost. 1999;82:92-94.

105. von Kummer R. Early major ischemic changes on computed tomography should preclude use of tissue plasminogen activator. Stroke. 2003;34:820-821.

106. Lyden P. Early major ischemic changes on computed tomography should not preclude use of tissue plasminogen activator. Stroke. 2003;34:821-822.

107. Roberts HC, Dillon WP, Furlan AJ, et al. Computed tomographic findings in patients undergoing intra-arterial thrombolysis for acute ischemic stroke due to middle cerebral artery occlusion: results from the PROACT II trial. Stroke. 2002;33:1565-1567.

108. Barber PA, Demchuk AM, Zhang J, et al. Validity and reliability of a quantitative computed tomography score in predicting outcome of hyperacute stroke before thrombolytic therapy. ASPECTS Study Group. Alberta Stroke Programme Early CT Score. Lancet. 2000;355:1670-1674.

109. Neumann-Haefelin T, Hoelig S, Berkefeld J, et al. Leukoaraiosis is a risk factor for symptomatic intracerebral hemorrhage after thrombolysis for acute stroke. Stroke. 2006;37:2463-2466.

110. Nighoghossian N, Hermier M, Adeleine P, et al. Old microbleeds are a potential risk factor for cerebral bleeding after ischemic stroke. A gradient-echo T2*-weighted brain MRI study. Stroke. 2002;33:735-742.

111. Fan YH, Zhang L, Lam WWM, et al. Cerebral microbleeds as a risk factor for subsequent intracerebral hemorrhages among patients with acute ischemic stroke. Stroke. 2003;34:2459-2462.

112. Chalela JA, Kang DW, Warach S. Multiple cerebral microbleeds: MRI marker of a diffuse hemorrhage-prone state. J Neuroimaging. 2004; 14:54-57.

113. Derex L, Nighoghossian N, Hermier M, et al. Thrombolysis for ischemic stroke in patients with old microbleeds on pretreatment MRI. Cerebrovasc Dis. 2004;17:238-241.

114. Kakuda W, Thijs VN, Lansberg MG, et al. Clinical importance of microbleeds in patients receiving IV thrombolysis. Neurology. 2005;65: $1175-1178$.

115. Fiehler J, Albers GW, Boulanger JM, et al. Bleeding risk analysis in stroke imaging before thromboLysis (BRASIL): pooled analysis of T2*-weighted magnetic resonance imaging data from 570 patients. Stroke. 2007;38:2738-2744. 
116. Thomalla G, Sobesky J, Köhrmann M, et al. Two tales: hemorrhagic transformation but not parenchymal hemorrhage after thrombolysis is related to severity and duration of ischemia. MRI study of acute stroke patients treated with intravenous tissue plasminogen activator within 6 hours. Stroke. 2007;38:313-318.

117. Trouillas P, Derex L, Philippeau F, et al. Early fibrinogen degradation coagulopathy is predictive of parenchymal hematomas in cerebral rt-PA thrombolysis. A study in 157 cases. Stroke. 2004;35:1323-1328.

118. Thomalla G, Schwark C, Sobesky J, et al. Outcome and symptomatic bleeding complications of intravenous thrombolysis within 6 hours in MRI-selected stroke patients. Comparison of a German multicenter study with the pooled data of ATLANTIS, ECASS, and NINDS tPA trials. Stroke. 2006;37:852-858.
119. Köhrmann M, Juttler E, Fiebach JB, et al. MRI versus CT-based thrombolysis treatment within and beyond the $3 \mathrm{~h}$ time window after stroke onset: a cohort study. Lancet Neurol. 2006;5:661-667.

120. Khatri P, Hill MD, Palesch YY, et al. Methodology of the Interventional Management of Stroke III Trial. Int J Stroke. 2008;3: $130-137$.

121. Lopez-Atalaya JP, Roussel BD, Levrat D, et al. Toward safer thrombolytic agents in stroke: molecular requirements for NMDA receptor-mediated neurotoxicity. J Cereb Blood Flow Metab. 2008; 28:1212-1221.

\section{Publish your work in this journal}

Vascular Health and Risk Management is an international, peerreviewed journal of therapeutics and risk management, focusing on concise rapid reporting of clinical studies on the processes involved in the maintenance of vascular health; the monitoring, prevention and treatment of vascular disease and its sequelae; and the involvement of metabolic disorders, particularly diabetes. This journal is indexed on PubMed Central and MedLine. The manuscript management system is completely online and includes a very quick and fair peer-review system, which is all easy to use. Visit http://www.dovepress.com/ testimonials.php to read real quotes from published authors.

Submit your manuscript here: http://www.dovepress.com/vascular-health-and-risk-management-journal 